\title{
What Does It Mean to Create Sustainable Science Curriculum Innovations? A Commentary
}

\author{
BARRY J. FISHMAN, JOSEPH KRAJCIK \\ School of Education, The University of Michigan, Ann Arbor, MI 48109-1259, USA
}

Received 12 April 2002; revised 8 July 2002; accepted 7 August 2002

\begin{abstract}
Two key issues for research on the creation of sustainable science curricula are the following: (1) How do we create curriculum innovations that will be used in classrooms after the developers or researchers depart; and (2) How do we create curriculum innovations that are scalable, such that teachers who never have direct contact with the developers may successfully enact them? We introduce the idea of usability as a guiding principle for the successful design of sustainable and scalable curricula, present a framework for examining usability in school contexts, and critique the ideas presented in this issue of Science Education using this framework. (C) 2003 Wiley Periodicals, Inc. Sci Ed 87:564-573, 2003; Published online in Wiley InterScience (www.interscience.wiley.com). DOI 10.1002/sce.10088
\end{abstract}

\section{INTRODUCTION}

The articles on creating sustainable curricula in this issue of Science Education come at a critical juncture for research in science education. The field as a whole has come to understand a great deal about how teachers teach and students learn science. The national science education standards documents (American Association for the Advancement of Science [AAAS], 1993; National Research Council, 1996) embody much of that knowledge, and challenge curriculum developers and teachers to make high-quality science learning materials and environments available to all students. Rather than seeking average student performance, an underlying principle of the standards movement is that all students should develop a deep understanding of science. The publication of the standards documents is partly in response to the overall movement in education toward systemic reform (Smith \& O'Day, 1991), wherein change is fostered by setting policy at the federal, state, and district levels and then tying those policies to stringent assessments (Goertz, 2001). It is then left up to schools at the local level to determine how they will respond to these directives and assessments. Frequently, the response is in the form of changes in local policies, increased professional development for teachers, and the acquisition of new materials or programs.

Correspondence to: Barry Fishman; e-mail: fishman@umich.edu

Contract grant sponsor: REPP, National Science Foundation.

Contract grant numbers: REC-9720383, REC-9725927, REC-9876150.

Contract grant sponsor: USI, National Science Foundation.

Contract grant number: ESR-9453665.

Contract grant sponsor: Spencer Foundation. 
Ironically, the high-stakes environment created by standards-based assessments often leads schools, particularly in urban settings, to employ teaching and learning approaches that are the opposite of what the standards intended, such as drill-and-practice and recitation. The curriculum programs described in this issue, on the other hand, begin with a strong focus on the central principles of the standards. Songer, Lee, and McDonald (this issue) argue that, "inquiry is at the core of what it means to be scientifically literate." This is important, because creating science curricula for widespread use that conform to the standards has proven to be nontrivial. The AAAS examined the major texts currently used by U.S. public schools. The results were less than positive. Of the 20 science textbooks (10 middle school and 10 high school Biology) evaluated, only one was found to be satisfactory in terms of having "a high potential for helping students learn ideas that are essential for ... science literacy" (Roseman, Kulm, \& Shuttleworth, 2001). Among the criteria the AAAS uses to evaluate textbooks are (a) that they take account of student ideas, (b) that they engage students with relevant contexts, experiences, and phenomena, and (c) that they promote student thinking about phenomena, experiences, and knowledge. Central to their analysis is that the criteria support students meeting learning objectives as stated in the standards. Clearly, it is no simple matter to create high-quality curriculum innovations that promote inquiry in accordance with national standards. It is, therefore, refreshing to see the attention of some of the most innovative research groups in science education turn their attention to this problem.

\section{THE BOTTOM LINE}

To frame our commentary on the articles in this issue, we begin by visiting what we see as the "bottom line" issues for research in the area of creating sustainable science curricula. From our perspective, two considerations are key:

1. How do we create science curriculum innovations that are sustainable, i.e., they will be used in classrooms after the developers or researchers have left the scene?

2. How do we create science curriculum innovations that are scalable, i.e., they can be used in places that have never had any contact or only minimal contact with the developers or researchers?

Cutting across these two issues is the notion of "usability." Usability is a concept that comes from the field of human-computer interaction, and denotes the ease (or lack thereof) with which the users of computers can take up and operate software (Soloway, Guzdial, \& Hay, 1994). We have appropriated this term in our own work (Blumenfeld et al., 2000) to describe the challenges that teachers and school organizations face in adopting curricular innovations of the sort described in these articles. If an innovation is "usable," this means three things: (1) that the innovation is adaptable to the organization's context, (2) that the organization is able to enact the innovation successfully, and (3) that the organization is able to sustain the innovation. If an innovation falls short in any of these areas, it indicates a serious usability problem.

\section{Just What Is a Curricular Innovation?}

Just what is meant when we call something a curricular innovation in science education? You might notice that in the earlier paragraph, the term "curricular innovation" is used, whereas throughout this issue the authors refer to curriculum materials. This subtle shift in terminology highlights one of the most important points we wish to make in this commentary - the challenge of creating sustainable standards-based learning environments 
is about much more than the curriculum materials alone. It is also about creating sustainable and scalable programs of professional development to help teachers use materials (Fishman et al., 2001); it is about fostering a management climate within the school that supports the uses of technology in addition to other learning tools of the curriculum (Fishman \& Gomez, 2000); and as is pointed out by the authors throughout this issue, it is about understanding and aligning the goals and affordances of the curriculum with the goals and capabilities of local settings. Furthermore, we would like to highlight the special nature of the curricular innovations that the authors in this issue address. Because they are attempting to help students pursue the inquiry goals of national standards, to foster deep learning and understanding on the part of students through constructivist approaches, we would like to refer to them as "cognitively oriented" curricular innovations.

\section{How Cognitively Oriented Curricular Innovations Are Developed}

The nature of research conducted on cognitively oriented curricular innovations is relevant to the challenge of making them sustainable and scalable, as Hickey (this issue) points out. Much of this research, especially where technology is involved, follows an approach in the spirit of design experiments (Brown, 1992). In these cases, the curriculum enactment is often supported by teams of faculty, graduate student researchers, extra technical assistance, and other infusions of resources necessary to make the curriculum work. Through these efforts, researchers create a context in which curriculum innovations are usable, enabling the study teaching and learning in these environments. Design experiments proceed iteratively, with successive enactments in which components of the innovation are varied in order to increase the overall performance of the innovation. A mixture of qualitative and quantitative approaches is used to document and analyze the performance of the curriculum. A great number of ideas related to the development of cognitively oriented science curricular innovations have been created in this way.

A side effect of the design research process is that researchers sometimes create environments that are not true to the larger, everyday context of schooling. Curricular innovations might be usable in the well-supported research environment, but are not usable by classrooms in general. Too frequently, following the initial research on the innovation, researchers leave unexamined future implementations, which are treated as "just implementation," something that happens after real research. This notion needs to be turned on its head, and the process of implementation must become the subject of serious research (c.f., Confrey, Castro-Filho, $\&$ Wilhelm, 2000) if we are to make traction on the problems of developing sustainable curricula. This is just what the authors in this issue attempt to do.

\section{A Framework for Considering the Usability of Cognitively Oriented Curricular Innovations}

In our own work with the Center for Learning Technologies in Urban Schools (LeTUS), we have developed a framework for considering the usability of any innovation in light of the capacity of the adopting context. To accomplish this, we array the elements of school culture, capability, and policy and management in the form of three axes originating from a common point (Blumenfeld et al., 2000; see Figure 1).

Any curricular (or other) innovation can be placed in the space created by these three axes, where the "distance" between the innovation and the origin represents the gap that exists between the capacity required to successfully use the innovation and the current capacity of the district. The creation of usable inquiry-based science curricula, conceptualized in this manner, is a process of working to "close the gaps" that exist. This might be a process 


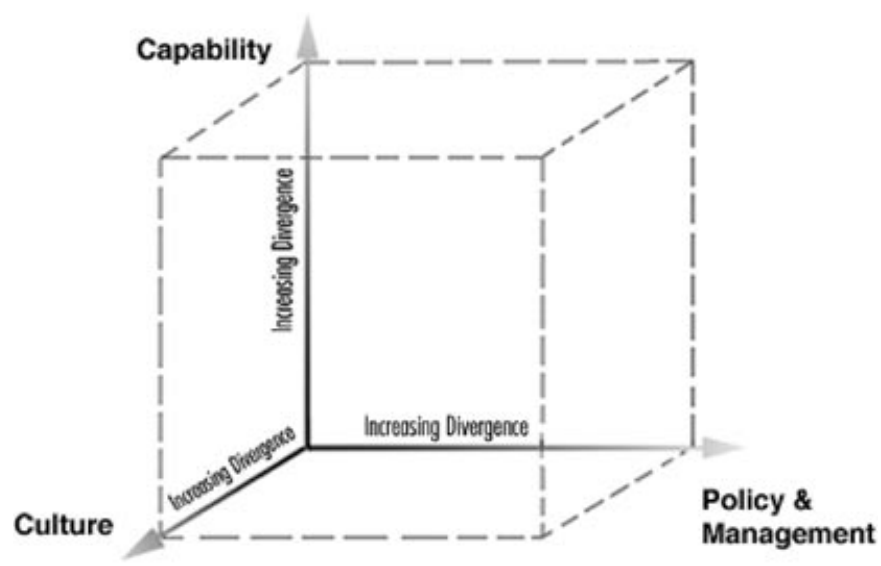

Figure 1. Diagnostic tool to identify usability challenges for innovations.

of increasing district or teacher capacity through professional development or changes in policy, or it might involve reworking the curricular innovation so that it better meets existing capacity. We argue that both are ultimately required in the creation of sustainable cognitively oriented science curriculum innovations. Learning how to "close the gaps" presents a formidable research challenge, one that necessitates asking new kinds of questions of just the kind that the authors of the papers in this volume take on. We also wish to point out that although this model was developed with educational systems in mind, the cube can be applied equally well to considering the success of innovations in a single classroom. We also want to stress again that the process of closing gaps in usability is a two-way street requiring changes both on the part of the curriculum developer and on the part of the school.

We now turn our attention directly to the ideas and arguments contained in the papers. As we examine the claims of each paper, we will turn back to the framework for considering usability in order to understand how each project considers the dimensions of school capacity in the development of their curricular innovations.

\section{A PROMISING START ON A CHALLENGING PROBLEM}

These papers do an admirable job of introducing the challenges inherent in developing usable inquiry-oriented curriculum innovations. Each paper makes headway on this problem, though each also raises many new questions, as is appropriate to exploratory research in the early phases of any new endeavor.

\section{Flexibly Adaptive Curricula}

Adaptability is a primary mechanism identified across these papers to foster sustainability. Squire et al. (this issue) argue that since all classrooms are unique, it is ultimately the responsibility of the teacher to adapt curriculum materials to fit both their own strengths, needs, and goals, and also the goals and needs of their students. The idea that teachers get "final say" in the design of the curriculum is an argument taken up by critics of "teacherproofing" (Gomez, Fishman, \& Pea, 1998), recognizing that such endeavors are ill-fated, particularly when attempting challenging instructional approaches such as inquiry. Squire et al. correctly point out that the burden lies with the developers of the curriculum to consciously increase the flexibility of the curriculum in order to encourage ownership and 
reinvention by its adopters. This is another way of expressing the need to reduce gaps in usability between an innovation and its intended adopters.

The concept of flexibly adaptive curriculum evokes Cohen and Ball's discussion of specification and development (Cohen \& Ball, 1999). Specification is the explicitness with which an innovation is expressed; some innovations consist mainly of goal statements that suggest a general direction but little more. More highly specified innovations are clear about curriculum, intended teaching practices, and desired learning goals. Development refers to the provision of resources required to enact innovations such as curriculum materials, professional development, and examples of teaching practice that can be educative for others. Specification without development requires teachers to figure out how to enact the innovation in their local setting, which can be a barrier to all but the most capable teachers. Development without specification provides resources for improvement, but not a clear picture as to what goals are to be attained. Such is the case when new computers are purchased for a school, but with little connection to curricular or pedagogical goals (Fishman \& Pinkard, 2001). If an innovation is both highly specified and developed, Cohen and Ball (1999) predict that it might be instructionally effective, but it is less likely to be adopted, because it is less flexibly adaptive to the goals and needs of local teachers' contexts. Creating sustainable curriculum innovations requires careful balancing between specification and development in order to preserve adaptive flexibility without diminishing usability.

Squire et al. (this issue) examine the implementation of a technology-rich air quality curriculum in four different classroom settings that were chosen to represent a range of variables the investigators suspected would be meaningful with respect to the local interpretation of the curricular innovation. Their choice of study sites varied by grade level, demographics, and instructional orientation of the teachers and schools. Unpacking the meaning of differences at this level of analysis is important. In their analysis, Squire et al. uncovered information about the usability of their curricula along the dimensions of classroom culture and capability (see Figure 1). They conclude that when teachers are able to successfully reinvent the curriculum in their context (i.e., reduce gaps between the curriculum's demands and local capabilities and/or culture), the maximum success was found. Furthermore, they found that rather than imposing change on classrooms through their curricular designs, the "dominant classroom culture persisted" through the implementation of the curriculum in each case, effectively changing the curriculum innovation in a different way in each setting. This is sensible, as the adoption of any complex educational innovation should logically result in changes both in the context and in the innovation. Adaptation is a two-way street. Using Cohen and Ball's language (Cohen \& Ball, 1999), Squire et al.'s innovation can be seen as well specified in that there is a strong emphasis placed upon theories of learning and instruction. However, the innovation is not well developed, as it is left up to the classroom teacher to make local decisions about how to enact the ideas behind the curriculum. This does increase adaptive flexibility, but at a cost.

In their paper, Linn, Clark, and Slotta (this issue) also argue in favor of flexibly adaptive learning environments. Their Web Integrated Science Environment (WISE) is modular, allowing teachers to select materials that fit their needs. Furthermore, the WISE work is built on partnerships with museum, scientific, and cultural organizations that are likely to be of interest to teachers, helping to ground the curriculum in materials that manage to be innovative and familiar at the same time. Strengths of their model include a platformindependent web environment (reducing the need for specialized technology), and a reusable framework for their projects and activities that make it easier on the one hand for designers to create new materials, and easier on the other hand for teachers to make use of projects and activities once they have become familiar with the overall format. WISE is well developed in 
terms of the range of resources that are available for ready use. WISE is also well specified in terms of its philosophy of learning, as embodied in the Scaffolded Knowledge Integration framework (Linn \& Hsi, 2000).

WISE is the latest instantiation of Linn's model of inquiry-based science teaching and learning, taking its position in a long line of highly regarded design-based research efforts that include the Computer as Learning Partner (Linn \& Hsi, 2000) and the Knowledge Integration Environment (Bell, Davis, \& Linn, 1995) projects. As such, WISE has the benefit of building upon a strong base of empirically demonstrated successful materials and practices. This is important, because as Linn et al. (this issue) argue, a key feature of their innovation is that it is "empirically validated." It is hard to conduct research on the sustainability of materials when the materials themselves may still be in rapid flux, or potentially insufficient to the task for which they were intended. This is pointed out by Squire et al. (this issue) as a potential shortcoming of their own exploration.

Songer, Lee, and McDonald (this issue) come at the issue of adaptability from a different perspective, by arguing that curricula need to contain a broader variety of images of inquiry to represent different classroom contexts. This is an example of providing more development (Cohen \& Ball, 1999), but in a fashion that encourages flexibility through the use of multiple exemplars. By providing such images, the usability of the curriculum increases because teachers are able to find models and examples that resemble their own environment. An initial impediment to reform in many cases is the reaction from practitioners that "that won't work in my classroom because of ..." followed by statements about the abilities of the children, the background of families, the richness of the resource environment, and so forth (Elmore, 2000). The ideas championed by the Kids as Global Scientists (KGS) researchers will inevitably help to reduce this barrier.

\section{Audience Issues: For Whom Are We Designing?}

Songer, Lee, and McDonald (this issue) tackle crucial audience issues of research on inquiry-based science curricula head-on. They recognize that a shortcoming in our understanding of how to develop sustainable science curriculum materials lies not in the examination of the materials themselves so much as in an examination of the contexts in which we come to understand those materials. To that end, the KGS research calls for a broadening of the contexts in which we conduct research on inquiry-oriented science curricula and also a reconsideration of the kinds of research questions that we ask.

One of the most interesting aspects of the data from the KGS research is the ways in which the two groups of teachers depicted are similar and dissimilar. For instance, differences in the value placed upon self-paced instruction. This may be a function of the amount of guidance and structure that many urban teachers feel their students need. This reflects an instructional principle articulated by Delpit (1988) in her writing about the pedagogical needs of African American students, of whom KGS urban classrooms are largely comprised.

WISE faces a similar challenge at this point in its evolution. Linn, Clark, and Slotta (this issue) are now trying to determine how to bring their empirically validated curriculum innovation to ever larger numbers of teachers than were part of the intensive but small-scale design-based research partnerships of their past. So far, their partnership model has helped them develop robust materials. This creates a challenge in terms of scalability as they now try to include teachers who are not "part of the partnership." Having teachers as first-class members of that partnership is a real strength of the WISE model. Partnerships build the kind of trust that is necessary to negotiate gaps in usability (Blumenfeld et al., 2000). But will the input of those teachers who are in the partnership be sufficient to develop for the broad range of classrooms in the world that WISE hopes to reach? It is too early to tell at this point. It is 
important that future WISE partnerships provide linkages to broader populations of teachers, particularly the kinds of teachers that the KGS group describes with the label "urban."

As the field moves forward into the relatively uncharted waters of research on creating sustainable science curriculum innovations, we need to refine a language for describing our work with each other, in order to promote understanding of the challenges we encounter. Songer, Lee, and McDonald (this issue) struggle with terminology related to describing teachers who come to participate in their project through different means. The term "maverick" is used to describe those teachers who seek out KGS on their own, usually by finding it on the web. The term "urban" is used to describe those teachers who come to use KGS as a function of their participation in an NSF-funded Urban Systemic Program. The choice of these particular terms sets up an unfortunate dichotomy between "mavericks" and "urbans," one which is unfair to the latter. Certainly there are teachers with "maverick" characteristics in urban settings. This seems to be true even among the KGS maverick population. According to the authors, KGS was used in the focus year by 94 urban teachers. If there were 17 teachers from Detroit, then 77 non-Detroit urban teachers chose to participate in KGS by following the same path as the "mavericks." The Detroit teachers selected for inclusion in the reported study are described as "successful urban," implying that they possess some characteristics that set them apart from another group that is not included in the current research. In this way the authors appear to have operationalized a description of their current participants in creating these terms, as opposed to attempting to create higher level generalizable categories. In our own work (Bobrowsky, Marx, \& Fishman, 2001; Fishman, 2002), we have made a distinction between teachers who are "volunteers," implying the same sense of personal and professional qualities that Songer et al. use to describe "mavericks," and "nonvolunteers," meant to imply that teachers are directed to participate as a result of some other association. Our terms are not perfect either. We agree with Songer et al. (this issue) that these distinctions are important, and we call attention to this matter to urge the field to consider the importance of developing a common language to address the new issues arising out of research on sustainable and scalable curriculum development.

\section{Policy and Management Considerations}

After examining their data, the KGS team concludes that its inquiry-based curriculum is successful in diverse settings. In part, this was because the KGS team provided a level of support, including focused professional development, for the urban teachers in Detroit that far exceeded that provided to the "maverick" population of teachers using their curricula elsewhere. A key issue that remains is how to create curriculum innovations that are sustainable and usable by "urban" (or "nonvolunteer") teachers without such intensive support, or else superb curricula like that created by the KGS group will remain unavailable to all but a select group of teachers and their students. The primary achievement of the KGS research reported in this issue is the further refinement of our understanding of what differences among classrooms and teachers are worthy of attention.

Of the three projects reported in these pages, the KGS described by Songer, Lee, and McDonald (this issue) is the only one that actively considers the policy and management dimension of the usability framework we presented in Figure 1. This dimension of the framework is critically important to establishing the context that surrounds and supports classroom enactment. Squire et al. (this issue) leave it up to the teacher to interpret and negotiate the larger context. While the teacher is likely to be a key informant in helping the researchers to understand the limitations of the surrounding context, our experiences in Detroit (Blumenfeld et al., 2000) indicate that teachers do not have the influence to produce necessary changes within their own building (e.g., asking for scheduling changes to increase 
access to a shared computer lab), not to mention fostering changes at the district level (e.g., asking for increased network reliability or technical support). Similarly, by relying so far on volunteer or maverick teachers who already have the appropriate surrounding context, WISE has managed to avoid the need to work directly with school administrations. We believe that the KGS research strikes the proper note with their focus on these issues. A critical next step is to learn more about ways that researchers can collaborate with and influence the policy and management structure of school systems in order to increase the usability of science curricula, and thus its potential for scalability and sustainability.

\section{Key Methodological Issues}

Hickey (this issue) takes on one of the most daunting challenges facing research on sustainable science curriculum innovations: creating data about implementation that matches current policy and assessment frameworks. The particular problem that Hickey takes on is the challenge of generating such data in the context of the sociocultural research frameworks that design researchers so often favor. It is critical that in doing this work, we create data on student performance and learning that will be compelling to both policymakers and educators in an era of high-stakes testing. We must be able to present strong evidence that children learn when using cognitively oriented science curriculum innovations. This is a critical area in which many curricular innovations currently have huge usability gaps.

The importance of this question was raised several notches by the Bush administration's increased emphasis on annual testing of students in reading and math (U.S. Department of Education, 2001). This creates an environment where it can be difficult to get school and teacher support for engaging in standards-based inquiry projects in science when their attention needs to be devoted to raising test scores in other content areas such as reading and math. Another challenge comes from the recent federal emphasis on randomized experimental designs as the preferred method for educational research. This de-emphasizes the importance of the design-based research (e.g., Brown, 1992; Collins, 1999; Edelson, 2002) that is so important to the early phases of testing new curricular ideas, and will continue to be critical for exploring the adaptability of curriculum innovations across contexts, as each of the authors in this issue are attempting to do. Hickey is correct to emphasize the point that research in the creation of sustainable curriculum innovations must continue to provide compelling evidence for student learning through the use of those materials. All of the authors in this issue are cognizant of this problem, though their research designs vary in the extent to which they can currently address the challenge.

There will need to be other changes in the way that we conduct research as well if we are to ultimately succeed. First, as alluded to in our discussion of the usability framework, the adaptation of innovations for use in particular school contexts requires a great deal of collaboration, and therefore trust, between the school organization and the research organization (Blumenfeld et al., 2000). Establishing this level of trust requires a fundamental shift in the way that we position the research enterprise with respect to schools. Schools need to have joint ownership of curricular innovations (McMillan-Culp \& Honey, 2000), or else they will not recognize the need to create change in order to reduce usability gaps in capability, culture, or policy and management. Research organizations need to recognize that the expertise necessary to help school systems think about curricular adaptation systemically may be beyond the reach of any individual or small group of researchers. Partnerships within the academy, therefore, are necessary to foster this kind of work. The current 3- to 5-year funding cycles for research make these types of partnerships and collaborations difficult. Funding agencies need to rethink these cycles in order to facilitate change. As Hickey (this issue) points out, we do not necessarily need a new methodology, but we do need to figure 
out how to make our current methodologies work better to serve the needs we face in this new research environment.

\section{CONCLUSION}

The challenge of creating sustainable cognitively oriented science curriculum innovations is ultimately a challenge of increasing capacity, both of school systems and of research organizations. The papers in this set introduce a range of considerations in this arena, and advance ideas to move the agenda forward. In this commentary, we have added what we believe to be other critical considerations, such as the importance of viewing innovations as more than just materials, and the need to focus on the usability of innovations in terms of the fit between the demands they make upon their users and the capacity of the teachers and schools who hope to use them.

From our perspective, it seems clear that as a field we are only just beginning to uncover the issues involved in the creation of rich, inquiry-oriented science curriculum innovations that are truly scalable, sustainable, and ultimately usable in a broad range of settings. We concur with Hickey (this issue) that, as a field, we need to provide greater evidence that these innovations foster increased student learning. We hope that the field takes up the challenge presented in these papers and makes it a central focus for research in the coming decades.

The research described in this paper was funded with support from the National Science Foundation under the following programs: REPP (REC-9720383, REC-9725927, REC-9876150), and USI (ESR9453665). Additional funding was provided by the Spencer Foundation. Thanks also to our colleagues and students in the Center for Highly Interactive Computing in Education who make this work possible, particularly Phyllis Blumenfeld, Ron Marx, and Elliot Soloway. All opinions expressed in this work are the authors' and do not necessarily represent either the funding agencies or the University of Michigan.

\section{REFERENCES}

American Association for the Advancement of Science. (1993). Benchmarks for science literacy, Project 2061. New York: Oxford University Press.

Bell, P., Davis, E., \& Linn, M. C. (1995). The knowledge integration environment: Theory and design. In J. Schnase \& E. Cunnius (Eds.), Computer Support for Collaborative Learning (pp. 14-21). Bloomington, IN: Erlbaum.

Blumenfeld, P., Fishman, B. J., Krajcik, J., Marx, R. W., \& Soloway, E. (2000). Creating usable innovations in systemic reform: Scaling-up technology-embedded project-based science in urban schools. Educational Psychologist, 35(3), 149-164.

Bobrowsky, W., Marx, R., \& Fishman, B. (2001, March). The empirical base for professional development in science education: Moving beyond volunteers. Paper presented at the Annual Meeting of the National Association of Research in Science Teaching, St. Louis, MO.

Brown, A. L. (1992). Design experiments: Theoretical and methodological challenges in creating complex interventions in classroom settings. The Journal of the Learning Sciences, 2(2), 141-178.

Cohen, D. K., \& Ball, D. L. (1999). Instruction, capacity, and improvement (CPRE Research Report Series RR-043). Philadelphia, PA: University of Pennsylvania Consortium for Policy Research in Education.

Collins, A. (1999). The changing infrastructure of education research. In E. Lagemann \& L. Shulman (Eds.), Issues in education research (pp. 289-298). San Francisco: Jossey-Bass.

Confrey, J., Castro-Filho, J., \& Wilhelm, J. (2000). Implementation research as a means to link systemic reform and applied psychology in mathematics education. Educational Psychologist, 35(3), 179-191. 
Delpit, L. D. (1988). The silenced dialogue: Power and pedagogy in educating other people's children. Harvard Educational Review, 58(3), 280-298.

Edelson, D. C. (2002). Design research: What we learn when we engage in design. Journal of the Learning Sciences, 11(1), 105-121.

Elmore, R. F. (2000). Building a new structure for school leadership. Washington, DC: Albert Shanker Institute.

Fishman, B. (2002, April). Linking the learning sciences to systemic reform: Teacher learning, leadership \& technology. Paper presented at the Annual Meeting of the American Educational Research Association, New Orleans, LA.

Fishman, B., \& Gomez, L. (2000). New technologies and the challenge for school leadership. In M. Honey \& C. Shookhoff (Eds.), The Wingspread Conference on Technology's Role in Urban School Reform: Achieving Equity and Quality (pp. 13-21). Racine, WI: The Joyce Foundation, The Johnson Foundation, and the EDC Center for Children and Technology.

Fishman, B., \& Pinkard, N. (2001). Bringing urban schools into the information age: Planning for technology vs. technology planning. Journal of Educational Computing Research, 25(1), 63-80.

Goertz, M. E. (2001). Standards-based accountability: Horse trade or horse whip? In S. H. Fuhrman (Ed.), From the capitol to the classroom: Standards-based reform in the states. 100th Yearbook of the National Society for the Study of Education (Part II) (pp. 39-59). Chicago, IL: University of Chicago Press.

Gomez, L., Fishman, B., \& Pea, R. (1998). The CoVis Project: Building a large scale science education testbed. Interactive Learning Environments, 6(1/2), 59-92.

Linn, M. C., \& Hsi, S. (2000). Computers, teachers, peers. Mahwah, NJ: Erlbaum.

McMillan-Culp, K., \& Honey, M. (2000). Scale and localization: The challenge of implementing what works. In M. Honey \& C. Shookhoff (Eds.), The Wingspread conference on technology's role in urban school reform: Achieving equity and quality (pp. 41-46). Racine, WI: The Joyce Foundation, The Johnson Foundation, and the EDC Center for Children and Technology.

National Research Council. (1996). The national science education standards. Washington, DC: National Academy Press.

Roseman, J. E., Kulm, G., \& Shuttleworth, S. (2001). Putting textbooks to the test. ENC Focus, 8(3), $56-59$.

Smith, M. S., \& O'Day, J. (1991). Systemic school reform. In S. H. Fuhrman \& B. Malen (Eds.), The politics of curriculum and testing (pp. 233-267). New York: Falmer.

Soloway, E., Guzdial, M., \& Hay, K. (1994). Learner-centered design: The challenge for HCI in the 21 st century. Interactions, 1(2), 36-48.

U.S. Department of Education. (2001). No child left behind. Retrieved June 12, 2002, from the World Wide Web: http://www.NoChildLeftBehind.gov/. 